\title{
Increasing expression of serine protease matriptase in ovarian tumors: tissue microarray analysis of immunostaining score with clinicopathological parameters
}

\author{
Jong-Shiaw Jin ${ }^{1}$, Dar-Shih Hsieh ${ }^{2}$, Shih-Hurng Loh ${ }^{3}$, Ann Chen ${ }^{1}$, Chen-Wen Yao ${ }^{1}$ and
} Chung-Yang Yen ${ }^{2}$

${ }^{1}$ Department of Pathology, Tri-Service General Hospital, National Defense Medical Center, Taipei, Taiwan, ROC; ${ }^{2}$ Division of Urology, Department of Surgery, Tri-Service General Hospital, National Defense Medical Center, Taipei, Taiwan, ROC and ${ }^{3}$ Department of Pharmacology, National Defense Medical Center, Taipei,

Taiwan, ROC

\begin{abstract}
Matriptase is a type II transmembrane serine protease expressed by cells of surface epithelial origin, including epithelial ovarian tumor cells. Matriptase cleaves and activates proteins implicated in the progression of cancer and represents a potential prognostic and therapeutic target. The aim of this study was to examine the expression of matriptase in ovarian tumors and to assign clinicopathological correlations. Immunohistochemical analysis of matriptase was performed in tissue microarrays of 164 ovarian neoplasms including 84 serous adenocarcinomas, 23 mucinous adenocarcinomas, 10 endometrioid adenocarcinomas, six yolk sac tumors, 12 clear cell carcinomas, six dysgerminomas, eight granulosa cell tumors, four transitional cell carcinomas, five fibromas, and six Brenner tumors. All ovarian tumors except the fibromas and Brenner tumors showed significant expression of matriptase. The matriptase scores were significantly higher in the tumors than in their nontumor counterparts (304 \pm 26 for serous adenocarcinoma; $361 \pm 28$ for mucinous adenocarcinoma; $254 \pm 17$ for endometrioid adenocarcinoma; $205 \pm 19$ for yolk sac tumor; $162 \pm 16$ for clear cell carcinoma; $109 \pm 11$ for dysgerminoma; $105 \pm 9$ for granulosa cell tumor; and $226 \pm 18$ for transitional cell carcinoma). Matriptase scores in serous adenocarcinoma were correlated with TNM stage and FIGO stage. Our findings demonstrate for the first time that matriptase is overexpressed in many malignant ovarian tumors. It may be a novel biomarker for diagnosis and treatment of malignant ovarian tumors.
\end{abstract}

Modern Pathology (2006) 19, 447-452. doi:10.1038/modpathol.3800495; published online 20 January 2006

Keywords: serine protease; matriptase; ovarian tumor; serous adenocarcinoma

Escape of tumor cells outside the ovarian capsule accounts for a significant percentage of treatment failures in patients with ovarian malignancies. ${ }^{1-3}$ Identification of mechanisms promoting tumor cell invasion may help direct creation of new therapies that can arrest local invasion and metastatic spread of disease.

The serine protease matriptase was isolated as a type II transmembrane serine protease expressed in breast carcinoma ${ }^{4-6}$ and subsequently cloned, ${ }^{7}$ and the gene sequence was identified as ST14/SNC19. ${ }^{8}$

Correspondence: Dr J-S Jin, MD, PhD, Department of Pathology, Tri-Service General Hospital, National Defense Medical Center, No. 325, Sec. 2, Cheng-Gong Road, Taipei, Taiwan, ROC.

E-mail: jsjin@ndmctsgh.edu.tw

Received 15 June 2005; revised 12 August 2005; accepted 13

August 2005; published online 20 January 2006
Recent study in the prostate cancer cell line PC-3 also demonstrated the presence of a membrane-type serine protease, MT-SP1, which is identical to matriptase..$^{9}$ The gene sequence for matriptase has also been cloned as $T A D G-15$, a member of a series of tumor-associated genes, expressed in ovarian carcinomas. ${ }^{10,11}$

Latent urokinase plasminogen activator (uPA) and prohepatocyte growth factor (pro-HGF) are substrates for matriptase. ${ }^{12,13}$ Both substrates participate in neoplastic invasion, most notably in the plasminmediated remodeling of the tumor extracellular matrix. The binding of uPA to its receptor increases the proliferation of human ovarian cancer cells in vitro. ${ }^{14}$ Recent studies show that the active form of HGF stimulates the in vitro chemotaxis, motility, and proliferation of ovarian carcinoma cells. ${ }^{15,16}$ 
In the present study, we evaluated the expression of matriptase in 164 ovarian tumors using tissue microarrays. Immunohistochemistry studies in tissue microarray slides are more reliable than studies in individual slides because all tissues in each array slide are prepared and stained under the same conditions and are analyzed simultaneously. ${ }^{17}$ In a series of patients with ovarian tumors, we compared immunostaining scores for matriptase among different histological types of ovarian tumors. Our findings demonstrate for the first time that matriptase is overexpressed in many malignant ovarian tumors.

\section{Materials and methods}

Paraffin-embedded tumor tissues were retrieved and tissue microarray slides were constructed according to a previously published method. ${ }^{18}$ The tissue microarray slides consisted of samples from 164 patients with primary ovarian neoplasms including 84 with serous adenocarcinomas (mean age 57), 23 with mucinous adenocarcinomas (mean age 48), 10 with endometrioid adenocarcinomas (mean age 42), six with yolk sac tumors (mean age 22), 12 with clear cell carcinomas (mean age 48), six with dysgerminomas (mean age 12), eight with granulosa cell tumors (mean age 43), four with primary transitional cell carcinomas (mean age 51), five with fibromas (mean age 42), and six with Brenner tumors (mean age 57).

All tumors were pathologically staged according to the TNM and FIGO stage systems. Stage T1 was defined as a tumor limited to ovaries. Stage T2 was defined as a tumor involving one or both ovaries with pelvic extension. Stage T3 was defined as tumor involving one or both ovaries with microscopically confirmed peritoneal metastasis outside the pelvis.

\section{Immunohistochemistry}

Tissue microarray sections were dewaxed in xylene, rehydrated in alcohol, and immersed in 3\% hydrogen peroxide for $10 \mathrm{~min}$ to suppress endogenous peroxidase activity. Antigen retrieval was performed by heating $\left(100^{\circ} \mathrm{C}\right)$ each section for $30 \mathrm{~min}$ in $0.01 \mathrm{~mol} / \mathrm{l}$ sodium citrate buffer ( $\mathrm{pH}$ 6.0). After three rinses (each for $5 \mathrm{~min}$ ) in phosphate-buffered saline (PBS), sections were incubated for $2 \mathrm{~h}$ at room temperature with a rabbit anti-human matriptase/ ST14 antibody (1:100; BETHYL Laboratories, Montgomery, TX, USA) diluted in PBS. After three washes (each for $5 \mathrm{~min}$ ) in PBS, sections were incubated with horseradish peroxidase-labeled mouse anti-rabbit immunoglobulin (DAKO, Glostrup, Denmark) for $1 \mathrm{~h}$ at room temperature. After three additional washes, peroxidase activity was developed with diaminobenzidine at room temperature.
For evaluation of immunoreactivity and histological appearance, all tissue microarray experiments were repeated 3 times and the slides were examined and scored by two authors concurrently. The intensity of cytoplasmic and membranous immunostaining of tumor cells was scored on a scale of 0 (no staining) to 4 (strongest intensity), and the percentage of tumor cells with cytoplasmic or membranous staining at each intensity was estimated. The percent of cells (from 0 to 100) at each intensity was multiplied by the corresponding immunostaining intensity (from 0 to 4 ) to obtain an immunostaining score ranging from 0 to 400 .

\section{Statistical Analysis}

All results are expressed as mean \pm standard error of the mean (s.e.m.). The immunostaining score of matriptase for different types of ovarian tumors was compared with the score for normal ovarian fibrovascular tissue. Statistical analysis was performed using Student's $t$-test between groups. A $P$-value less than 0.05 was considered to be statistically significant. SigmaState software (Jandel Scientific, USA) was used to perform linear regression testing to analyze the relationship between matriptase immunostaining scores and clinicopathological parameters.

\section{Results}

\section{Matriptase Expression in Ovarian Tumors}

The histology of the hematoxylin and eosin-stained ovarian tumors, including serous adenocarcinoma, mucinous adenocarcinoma, clear cell carcinoma, transitional cell carcinoma, endometrioid adenocarcinoma, yolk sac tumor, Brenner tumor, granulosa cell tumor, and dysgerminoma was confirmed by two pathologists (Figure 1).

Among the 164 ovarian tumors, matriptase immunoreactivity was seen on the cell surface and in the cytoplasm of tumor cells, but not on surrounding stromal cells or in the extracellular matrix (Figure 2). Immunostaining scores for matriptase in ovarian tumors ranged from a high of $361 \pm 28$ for mucinous adenocarcinoma to a low of $2 \pm 1$ for Brenner tumor. Data are presented in Table 1. All 84 serous adenocarcinomas and 23 mucinous adenocarcinomas were strongly (intensity score greater than or equal to 3 ) and diffusely ( $>90 \%$ tumor cells) positive for matriptase. The matriptase scores in ovarian tumors were significantly higher than in their nontumor counterparts $(304 \pm 26$ for serous adenocarcinoma; $361 \pm 28$ for mucinous adenocarcinoma; $254 \pm 17$ for endometrioid adenocarcinoma; $205 \pm 19$ for yolk sac tumor; $162 \pm 16$ for clear cell carcinoma; $109 \pm 11$ for dysgerminoma; $105 \pm 9$ for granulosa cell tumor; and $226 \pm 18$ for transitional 

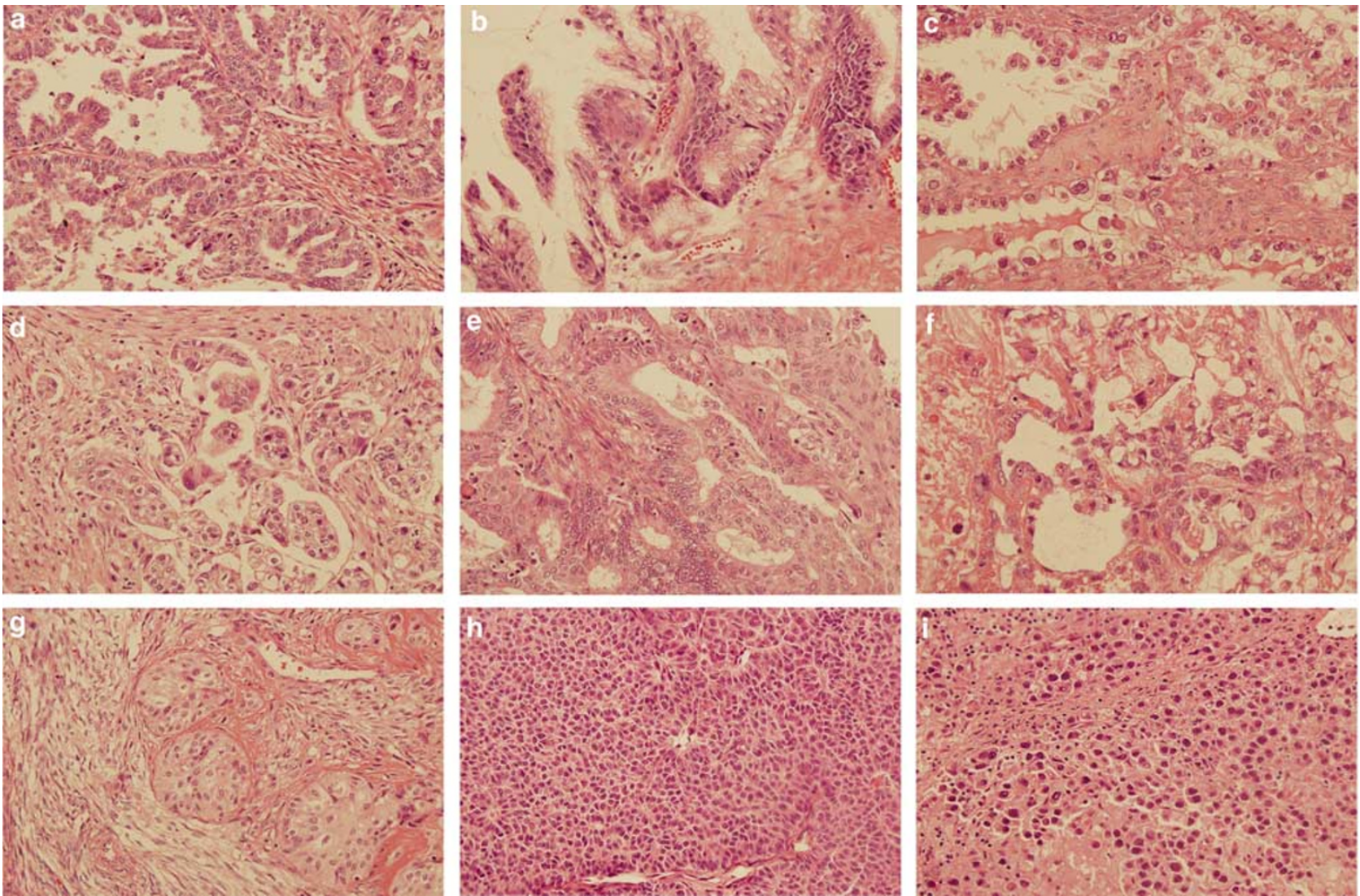

Figure 1 Hematoxylin and eosin staining of serous adenocarcinoma (a), mucinous adenocarcinoma (b), clear cell adenocarcinoma (c), transitional cell carcinoma (d), endometrioid adenocarcinoma (e), yolk sac tumor (f), Brenner tumor (g), granulosa cell tumor (h), and dysgerminoma (i). Original magnification $\times 400$.

cell carcinoma). Expression of matriptase in fibroma and Brenner tumor was not increased.

\section{Correlation of Matriptase Immunostaining Scores with Clinicopathological Features}

In Figure 3, there were 40 cases of stage T1, 34 cases of stage T2, and 10 cases of stage T3 serous adenocarcinoma. Of the 84 cases of serous adenocarcinoma, 46 were stage No and 38 were stage N1. The immunostaining scores for matriptase in serous adenocarcinoma showed significant correlation with TNM stage and FIGO stage. However, there were no significant relationships between matriptase immunostaining scores and clinicopathological features in mucinous adenocarcinoma, endometrioid adenocarcinoma, yolk sac tumor, clear cell carcinoma, dysgerminoma, granulosa cell tumor, and transitional cell carcinoma (data not shown).

\section{Discussion}

The human gene for matriptase is located within chromosome 11q24-q25. ${ }^{8}$ Loss of heterozygosity $(\mathrm{LOH})$ in this chromosome 11 region has been shown in ovarian epithelial cancers including serous adenocarcinoma and mucinous adenocarcinoma, ${ }^{19-21}$ and LOH of $11 \mathrm{q} 24-\mathrm{q} 25$ has been associated with poor survival of patients with ovarian epithelial cancers. ${ }^{19,20}$ Although frequent $\mathrm{LOH}$ of 11q24-q25 has also been identified in colorectal carcinoma, no correlation with patient's survival was found. ${ }^{22} \mathrm{LOH}$ of $11 \mathrm{q} 22-24$ in cervical carcinomas and LOH of 11q22-11qter in breast cancers have also been observed and these findings suggest that this region may harbor a tumor-associated gene common to many types of human carcinomas. ${ }^{23-25}$

In fact, because of matriptase's ability to cleave and activate growth factors and other serine proteases thought to be involved in cancer progression, matriptase has been implicated as a potential proinvasion and metastasis factor. In support of this hypothesis, our results demonstrated that significantly elevated matriptase immunostaining scores were found in serous adenocarcinoma, mucinous adenocarcinoma, endometrioid adenocarcinoma, yolk sac tumor, clear cell carcinoma, dysgerminoma, granulosa cell tumor, and transitional cell carcinoma. This observation may suggest that matriptase has an important role in the clinical aggressiveness of these malignant ovarian tumors.

Matriptase immunostaining scores in serous adenocarcinoma were significantly correlated with 

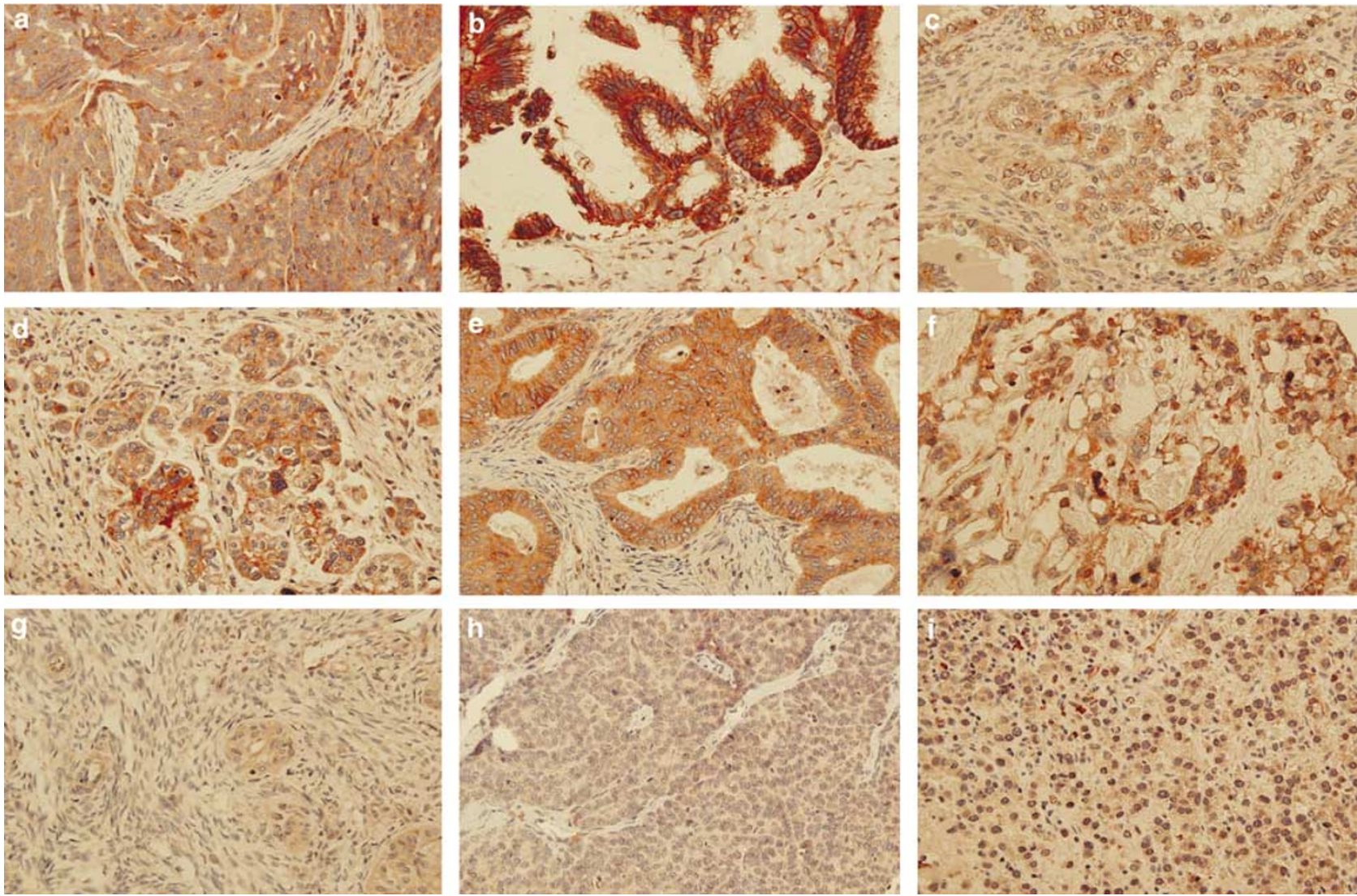

Figure 2 Immunohistochemical analysis of serous adenocarcinoma (a), mucinous adenocarcinoma (b), clear cell adenocarcinoma (c), transitional cell carcinoma (d), endometrioid adenocarcinoma (e), yolk sac tumor (f), Brenner tumor (g), granulosa cell tumor (h), and dysgerminoma (i) with anti-matriptase antibody. Original magnification $\times 400$.

Table 1 Immunostaining scores for matriptase in different ovarian tumors

\begin{tabular}{lccc}
\hline Ovarian tumor & \% Staining & Intensity & Total score \\
\hline Serous adenoCA $(n=84)$ & $94 \pm 54$ & $3.1 \pm 0.3$ & $304 \pm 26^{*}$ \\
Mucinous adenoCA $(n=23)$ & $96 \pm 6$ & $3.7 \pm 0.5$ & $361 \pm 28^{*}$ \\
Endometrioid adenoCA $(n=10)$ & $92 \pm 7$ & $2.8 \pm 0.4$ & $2.4 \pm 0.4$ \\
YST $(n=6)$ & $84 \pm 4$ & $1.9 \pm 0.4$ & $1.4 \pm 0.3$ \\
Clear cell CA $(n=12)$ & $83 \pm 6$ & $1.1 \pm 0.2$ & $17^{*}$ \\
Dysgerminoma $(n=6)$ & $73 \pm 5$ & $2.9 \pm 0.5$ & $19^{*}$ \\
GCT $(n=8)$ & $91 \pm 6$ & $0.2 \pm 0.3$ & $109 \pm 16^{*}$ \\
TCC $(n=4)$ & $79 \pm 7$ & $0.3 \pm 0.2$ & $226 \pm 18^{*}$ \\
Fibroma $(n=5)$ & $8 \pm 5$ & $0.2 \pm 0.2$ & $2 \pm 2$ \\
Brenner tumor $(n=6)$ & $6 \pm 3$ & $5 \pm 3$ & $2 \pm 1$ \\
Nontumor part $(n=164)$ & $5 \pm 1$ \\
\hline
\end{tabular}

Data are means \pm standard error of the mean (s.e.m.) of immunostaining score for matriptase in ovarian tumors and nontumor ovarian fibrovascular tissue.

${ }^{*} P<0.05$ vs nontumor ovarian fibrovascular tissue.

Serous adenocarcinoma, serous adenoCA; mucinous adenocarcinoma, mucinous adenoCA; endometrioid adenocarcinoma, endometrioid adenoCA; yolk sac tumor, YST; clear cell carcinoma, clear cell CA; granulosa cell tumor, GCT; transitional cell carcinoma, TCC.

the TNM stage and FIGO stage. However, there were no significant correlations between matriptase immunostaining scores and clinicopathological features in mucinous adenocarcinoma, endometrioid adenocarcinoma, yolk sac tumor, clear cell carcinoma, dysgerminoma, granulosa cell tumor, and transitional cell carcinoma. Lack of correlation may due to the limited number of cases in our study; future efforts will be directed at confirming matriptase's role in tumor progression and assessing the mechanism by which it contributes to the other invasive phenotypes of ovarian tumors. 

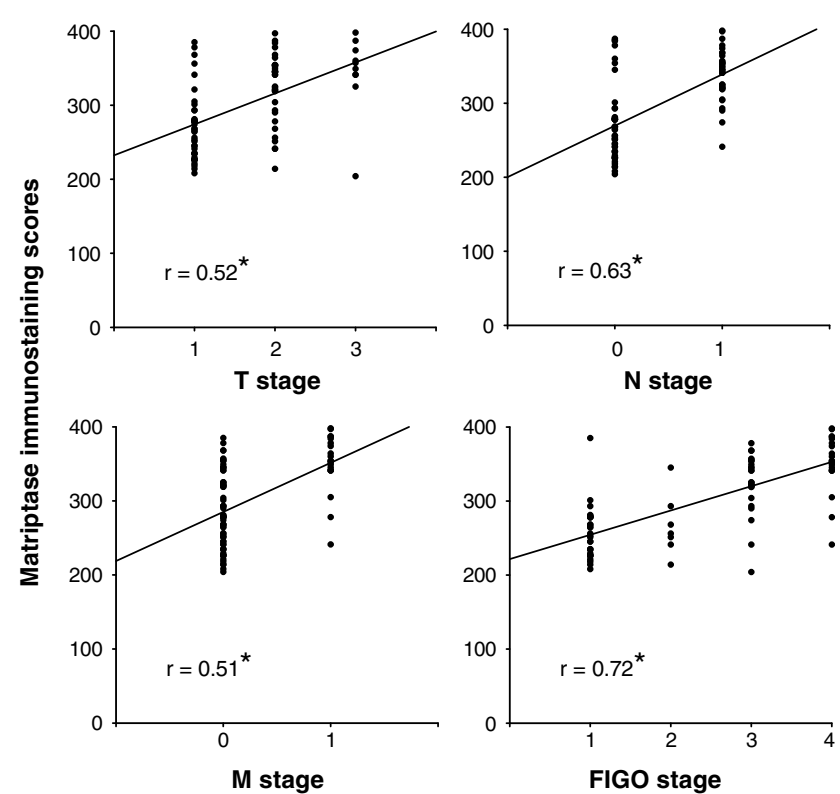

Figure 3 Clinicopathological correlations with matriptase immunostaining scores in serous adenocarcinoma. ${ }^{*}$ indicates statistical significance of linear regression testing $(P<0.05)$.

Recent advance in the tissue microarray technique has created a powerful tool for evaluating tumors simultaneously with histological and immunohistochemical analyses. ${ }^{17-28}$ Previous studies of individual cases limited the value of stain intensity-based immunohistochemical quantitation because of the instability of the signal generated by chemical reaction under different environmental conditions. ${ }^{17,26-28}$ However, immunohistochemistry studies using tissue microarray technique are more reliable than studies involving individual tissues because all tissues are processed under the same immunostaining conditions and analyzed simultaneously in a tissue microarray slide. ${ }^{17,26-28}$ Thus, the immunostaining scores used in our study are a better reflection of the relative level of matriptase protein expression in tumors.

In conclusion, we demonstrated for the first time that matriptase is expressed in many malignant ovarian tumors, except fibroma and Brenner tumor. Increased expression of matriptase in serous adenocarcinoma has significant clinicopathological relationships to the aggressiveness seen with this tumor. Our results add considerable support to the likelihood that matriptase acts as a novel biomarker for diagnosis and treatment of malignant ovarian tumors.

\section{Acknowledgements}

We thank Mr Yu-Ting Chen for his excellent technical assistance. This study was supported by grants from National Science Counsel, NSC94-2320B-016-017, and Tri-Service General Hospital, TSGHC94-112, Taiwan, ROC.

\section{References}

1 Piek JM, Kenemans P, Verheijen RH. Intraperitoneal serous adenocarcinoma: a critical appraisal of three hypotheses on its cause. Am J Obstet Gynecol 2004; 191:718-732.

2 Brown E, Stewart M, Rye T, et al. Carcinosarcoma of the ovary: 19 years of prospective data from a single center. Cancer 2004;100:2148-2153.

3 Itamochi H, Kigawa J, Sugiyama $\mathrm{T}$, et al. Low proliferation activity may be associated with chemoresistance in clear cell carcinoma of the ovary. Obstet Gynecol 2002;100:281-287.

4 Shi YE, Torri J, Yieh L, et al. Identification and characterization of a novel matrix-degrading protease from hormone-dependent human breast cancer cells. Cancer Res 1993;53:1409-1415.

5 Lin CY, Wang JK, Torri J, et al. Characterization of a novel, membrane-bound, $80-\mathrm{kDa}$ matrix-degrading protease from human breast cancer cells. Monoclonal antibody production, isolation, and localization. J Biol Chem 1997;272:9147-9152.

6 Lin CY, Anders J, Johnson M, et al. Purification and characterization of a complex containing matriptase and a Kunitz-type serine protease inhibitor from human milk. J Biol Chem 1999;274:18237-18242.

7 Lin CY, Anders J, Johnson M, et al. Molecular cloning of cDNA for matriptase, a matrix-degrading serine protease with trypsin-like activity. J Biol Chem 1999; 274:18231-18236.

8 Zhang Y, Cai X, Schlegelberger B, et al. Assignment of human putative tumor suppressor genes ST13 (alias SNC6) and ST14 (alias SNC19) to human chromosome bands 22q13 and 11q24-q25 by in situ hybridization. Cytogenet Cell Genet 1998;83:56-57.

9 Takeuchi T, Shuman MA, Craik CS. Reverse biochemistry: use of macromolecular protease inhibitors to dissect complex biological processes and identify a membrane-type serine protease in epithelial cancer and normal tissue. Proc Natl Acad Sci USA 1999;96: 11054-11061.

10 Tanimoto H, Underwood LJ, Wang Y, et al. Ovarian tumor cells express a transmembrane serine protease: a potential candidate for early diagnosis and therapeutic intervention. Tumour Biol 2001;22:104-114.

11 Oberst M, Anders J, Xie B, et al. Matriptase and HAI-1 are expressed by normal and malignant epithelial cells in vitro and in vivo. Am J Pathol 2001;158:1301-1311.

12 Takeuchi T, Harris JL, Huang W, et al. Cellular localization of membrane-type serine protease 1 and identification of protease-activated receptor- 2 and single-chain urokinase-type plasminogen activator as substrates. J Biol Chem 2000;275:26333-26342.

13 Lee SL, Dickson RB, Lin CY. Activation of hepatocyte growth factor and urokinase/plasminogen activator by matriptase, an epithelial membrane serine protease. J Biol Chem 2000;275:36720-36725.

14 Fischer K, Lutz V, Wilhelm O, et al. Urokinase induces proliferation of human ovarian cancer cells: characterization of structural elements required for growth factor function. FEBS Lett 1998;438:101-105.

15 Corps AN, Sowter HM, Smith SK. Hepatocyte growth factor stimulates motility, chemotaxis and mitogenesis in ovarian carcinoma cells expressing high levels of c-met. Int J Cancer 1997;73:151-155.

16 Sowter HM, Corps AN, Smith SK. Hepatocyte growth factor (HGF) in ovarian epithelial tumour fluids 
stimulates the migration of ovarian carcinoma cells. Int J Cancer 1999;83:476-480.

17 Lam JS, Belldegrun AS, Figlin RA. Tissue array-based predictions of pathobiology, prognosis and response to treatment for renal cell carcinoma therapy. Clin Cancer Res 2004;10:6304s-6309s.

18 Pan CC, Chen PCH, Chiang H. An easy method for manual construction of high-density tissue arrays. Appl Immunohistochem Mol Morphol 2004;12: 370-372.

19 Gabra H, Taylor L, Cohen BB, et al. Chromosome 11 allele imbalance and clinicopathological correlates in ovarian tumours. Br J Cancer 1995;72:367-375.

20 Gabra H, Watson JE, Taylor KJ, et al. Definition and refinement of a region of loss of heterozygosity at 11q23.3-q24.3 in epithelial ovarian cancer associated with poor prognosis. Cancer Res 1996;56:950-954.

21 Davis M, Hitchcock A, Foulkes WD, et al. Refinement of two chromosome 11q regions of loss of heterozygosity in ovarian cancer. Cancer Res 1996;56:741-744.

22 Connolly KC, Gabra H, Millwater CJ, et al. Identification of a region of frequent loss of heterozygosity at 11q24 in colorectal cancer. Cancer Res 1999;59: 2806-2809.
23 Gudmundsson J, Barkardottir RB, Eiriksdottir G, et al. Loss of heterozygosity at chromosome 11 in breast cancer: association of prognostic factors with genetic alterations. Br J Cancer 1995;72:696-701.

24 Gentile M, Olsen K, Dufmats M, et al. Frequent allelic losses at 11q24.1-q25 in young women with breast cancer: association with poor survival. Br J Cancer 1999;80:843-849.

25 Hampton GM, Penny LA, Baergen RN, et al. Loss of heterozygosity in cervical carcinoma: subchromosomal localization of a putative tumor-suppressor gene to chromosome 11q22-q24. Proc Natl Acad Sci USA 1994;91:6953-6957.

26 Kim HL, Seligson D, Liu X, et al. Using tumor markers to predict the survival of patients with metastatic renal cell carcinoma. J Urol 2005;173:1496-1501.

27 Bhattacharjee A, Richards WG, Staunton J, et al. Classification of human lung carcinomas by mRNA expression profiling reveals distinct adenocarcinoma subclasses. Proc Natl Acad Sci USA 2001;98:13790-13795.

28 Takahashi M, Rhodes DR, Furge KA, et al. Gene expression profiling of clear cell renal cell carcinoma: gene identification and prognostic classification. Proc Natl Acad Sci USA 2001;98:9754-9759. 